\title{
The Role of Rare Variants in Systolic Blood Pressure: Analysis of ExomeChip Data in HyperGEN African Americans
}

\author{
Yun Ju Sung ${ }^{a}$ Jacob Basson ${ }^{a}$ Nuo Cheng ${ }^{a}$ Khanh-Dung H. Nguyen ${ }^{c}$ \\ Priyanka Nandakumar ${ }^{c}$ Steven C. Hunt ${ }^{d}$ Donna K. Arnett ${ }^{\mathrm{e}}$ \\ Victor G. Dávila-Román ${ }^{b}$ Dabeeru C. Rao ${ }^{a}$ Aravinda Chakravartic ${ }^{c}$ \\ ${ }^{a}$ Division of Biostatistics, and ${ }^{b}$ Cardiovascular Division, Department of Medicine, Washington University School \\ of Medicine in St. Louis, St. Louis, Mo., ' Institute of Genetic Medicine, Johns Hopkins University School of \\ Medicine, Baltimore, Md., ${ }^{d}$ Cardiovascular Genetics, University of Utah, Salt Lake City, Utah, and e Department of \\ Epidemiology, School of Public Health, University of Alabama at Birmingham, Birmingham, Ala., USA
}

\section{Key Words}

Rare variants · ExomeChip · Gene-based analysis · Burden tests $\cdot$ SKAT $\cdot$ Systolic blood pressure $\cdot$ Family studies

\section{Abstract \\ Cardiovascular diseases are among the most significant health problems in the United States today, with their major risk factor, hypertension, disproportionately affecting Afri- can Americans (AAs). Although GWAS have identified doz- ens of common variants associated with blood pressure (BP) and hypertension in European Americans, these variants col- lectively explain $<2.5 \%$ of BP variance, and most of the ge- netic variants remain yet to be identified. Here, we report the results from rare-variant analysis of systolic BP using 94,595 rare and low-frequency variants (minor allele frequency, $M A F,<5 \%)$ from the Illumina exome array genotyped in 2,045 HyperGEN AAs. In addition to single-variant analysis, 4 gene-level association tests were used for analysis: burden and family-based SKAT tests using MAF cutoffs of 1 and $5 \%$. The gene-based methods often provided lower $p$ values than the single-variant approach. Some consistency was ob-}

served across these 4 gene-based analysis options. While neither the gene-based analyses nor the single-variant analysis produced genome-wide significant results, the top signals, which had supporting evidence from multiple genebased methods, were of borderline significance. Though additional molecular validations are required, 6 of the 16 most promising genes are biologically plausible with physiological connections to BP regulation.

(c) 2015 S. Karger AG, Basel

\section{Introduction}

Cardiovascular diseases are among the most significant health problems in the United States today, with hypertension affecting African Americans (AAs) disproportionately. Blood pressure (BP) and hypertension have been challenging phenotypes for gene discovery due to their complex nature. Although GWAS have successfully identified thousands of loci affecting complex human diseases, these identified loci only explain small proportions of heritability. For example, 3 recent GWAS consortia 
[1-3] identified dozens of common variants associated with BP and hypertension in European Americans. However, these variants collectively explain $<2.5 \%$ of $\mathrm{BP}$ variance, and most of the genetic variants remain yet to be identified [4]. The missing heritability might be partially explained by rare and low-frequency variants, with minor allele frequencies (MAF) $<5 \%$ [5].

Because the power to detect individual rare variants is limited by their low frequency, the development of novel statistical approaches for the analysis of rare variants has been an active area of research. These methods are broadly classified as burden and non-burden tests. Burden tests collapse rare variants in a genomic region (typically a gene) into a single burden variable to test for the combined effects of all rare variants in the region or gene. Such tests include the cohort allelic sum test (CAST) [6], the combined multivariate and collapsing $(\mathrm{CMC})$ method [7], the group-wise weighted sum test [8], and the proportion and presence tests by Morris and Zeggini [9]. Since these burden tests implicitly assume that all variants influence the trait in the same direction and with the same magnitude of effect, their power is reduced if their effects are not homogeneous [10]. Several non-burden tests have been developed that are less sensitive to heterogeneous effects [11-13]. Two one-sided statistics proposed by Ionita-Laza et al. [11] quantify enrichment in risk variants and protective variants. The $\mathrm{C}$-alpha test uses the distribution of genetic variation observed in cases and controls, maintaining consistent power when the target set contains both risk and protective variants [12]. The sequence kernel association test (SKAT) provides a flexible and computationally efficient approach using a scorebased variance component test and is applicable to both continuous and discrete traits [13]. Using the framework of linear mixed effect models, SKAT has been recently extended to handle family data, which is called the familybased SKAT (famSKAT) [14].

Here, we report the results from rare-variant analysis using 94,595 rare and low-frequency variants from the Illumina exome array (ExomeChip) genotyped in HyperGEN AAs. We performed 4 gene-level association analyses of systolic BP (SBP): burden and famSKAT tests with MAF cutoffs of 1 and 5\% accounting for family correlation. To account for population stratification in our AA subjects, we used principal components (PCs) of the common variants (with $\mathrm{MAF} \geq 5 \%$ ) on the ExomeChip as covariates in our analysis. A varying number of PCs (no PC, first 2 PCs and first 10 PCs) were used to evaluate the impact of population stratification on the analysis of rare variants.

Rare-Variant Analysis of SBP

\section{Methods}

\section{Study Sample}

We studied AAs in the Hypertension Genetic Epidemiology Network (HyperGEN) from the Family Blood Pressure Program [15]. The study recruited AA and European American participants at 5 field centers to investigate the genetic causes of hypertension and related conditions. Study participants were 1 of 3 types: (1) individuals in hypertensive sibships with at least 2 siblings diagnosed with hypertension; (2) random subjects, who were agematched with hypertensive siblings, or (3) unmedicated adult offspring of 1 or more of the hypertensive siblings. The study obtained informed consent from all participants and approval from the appropriate institutional review boards.

\section{Genotype and Phenotype Data}

A total of 2,147 HyperGEN AAs were genotyped using the Illumina exome array that covers the genome-wide exome variants. Illumina's clustering algorithm (GenTrain version 1.0), as implemented in GenomeStudio, was used for genotype calling. To check the quality and consistency of the genotyping process, 19 blind duplicate samples were included. We removed these duplicate samples and corrected sample mix-ups and pedigree errors, resulting in 2,111 subjects. We removed monomorphic markers and SNPs with a missing rate $>5 \%$ or Hardy-Weinberg p value $<10^{-6}$ and removed any genotypes with a non-Mendelian pattern of inheritance. The number of variants after quality control and exclusions was 124,759 . There were 30,164 common variants (with MAF $\geq 5 \%$ ) and 94,595 low-frequency variants (with MAF $<5 \%$ ). There were also extremely rare variants including 15,017 singletons and 9,323 doubletons.

We included 2,045 subjects with genotype, phenotype and covariate information. SBP was measured (on a $\mathrm{mm} \mathrm{Hg}$ scale) with a DINAMAP model 1846 SX/P automated oscillometric device using a standard protocol. Briefly, after a 5 -min rest period, the resting BP was measured 6 times, with a 2-min gap between the measurements. The average of 3 SBP measurements was used for each subject. In all analyses, we added $15 \mathrm{~mm} \mathrm{Hg}$ to the SBP values for subjects taking antihypertensive or BP-lowering medications. Age, sex, and body mass index were included as covariates. In addition, a varying number of PCs derived from common variants were included as covariates, as discussed below.

\section{Statistical Analysis}

We performed a single-variant analysis and the 4 sets of genome-wide gene-level association analysis. The latter are burden tests with MAF cutoffs of 1 and 5\% (Burden-1 and Burden-5) and famSKAT tests with MAF cutoffs of 1 and $5 \%$ (famSKAT- 1 and famSKAT-5). We used the annotation file for the Illumina exome array data, available from the CHARGE (Cohorts for Heart and Aging Research in Genetic Epidemiology) study [16]. For the single-variant analysis, we excluded singletons because false positive results may arise from an influential phenotype value of one subject. For the gene-level analyses, we excluded genes with only one variant as their results were identical to those of the single-variant analysis. We included singletons for the gene-level analyses. For example, when a gene included multiple singletons, all these singletons were jointly used for our gene-based analyses.

To perform the burden tests in HyperGEN AAs, we extended the burden test by Morris and Zeggini [9] to account for family 
Table 1. Genomic inflation values $(\lambda)$ for the 5 genome-wide analysis options using each of PC adjustments

\begin{tabular}{rlllll}
\hline PCs & Single-variant analysis & Burden-1 & Burden-5 & famSKAT-1 & famSKAT-5 \\
\hline 0 & 1.002 & 1.028 & 1.007 & 1.075 & 1.094 \\
2 & 0.999 & 1.025 & 1.006 & 1.079 & 1.102 \\
10 & 0.999 & 1.041 & 1.010 & 1.107 & 1.119 \\
\hline
\end{tabular}

Quantile-quantile plots are shown in online supplementary figure 1.

Table 2. The 11 top low-frequency variants from the single-variant analysis with a $\mathrm{p}$ value $<6 \times 10^{-5}$ (all these variants were exonic nonsynonymous)

\begin{tabular}{llrrllrll}
\hline Rank & SNP & Chr & \multicolumn{1}{l}{ BP } & Gene & MAF & Beta & SE & p \\
\hline 1 & rs138085317 & 4 & $114,290,810$ & ANK2 & 0.0042 & 27.3 & 5.6 & $1.2 \times 10^{-6}$ \\
2 & rs143104022 & 18 & $30,791,983$ & C180rf34 & 0.0012 & 43.3 & 9.9 & $1.2 \times 10^{-5}$ \\
3 & rs144164391 & 6 & $25,779,375$ & SLC17A4 & 0.0012 & 42.8 & 9.9 & $1.4 \times 10^{-5}$ \\
4 & rs75307540 & 11 & $6,452,889$ & HPX & 0.0093 & 15.9 & 3.8 & $2.9 \times 10^{-5}$ \\
5 & rs145926130 & 16 & $1,536,575$ & PTX4 & 0.0017 & 35.8 & 8.6 & $3.2 \times 10^{-5}$ \\
6 & rs35124934 & 2 & $3,744,990$ & ALLC & 0.0342 & 8.1 & 2.0 & $4.3 \times 10^{-5}$ \\
7 & rs6665210 & 1 & $145,532,823$ & ITGA10 & 0.0193 & -10.7 & 2.6 & $4.4 \times 10^{-5}$ \\
8 & rs75508009 & 5 & $111,519,758$ & EPB41L4A & 0.0022 & 30.2 & 7.4 & $4.5 \times 10^{-5}$ \\
9 & rs144714540 & 1 & $45,809,001$ & TOE1 & 0.0010 & 48.8 & 12 & $4.6 \times 10^{-5}$ \\
10 & rs139543898 & 1 & $158,389,863$ & OR10K2 & 0.0005 & 63.4 & 15.6 & $5.0 \times 10^{-5}$ \\
11 & rs146171474 & 4 & $122,833,191$ & TRPC3 & 0.0007 & 56 & 13.9 & $5.8 \times 10^{-5}$ \\
\hline
\end{tabular}

The genome-wide Bonferroni-corrected threshold corresponds to a p value $<6.4 \times 10^{-7}(=0.05 / 79,578)$. Boldfaced genes are biologically plausible, providing a physiological connection to BP regulation (as presented in the Discussion section). Except for TRPC3, which contained only 1 variant, these were included in table 3.

structure. For the combined effect of multiple rare variants in a genomic region, Morris and Zeggini [9] developed the burden test in a linear regression framework. For family data, a linear mixed model with a random polygenic component is commonly used to account for phenotypic correlations among related individuals. Therefore, we used a linear mixed effect modeling framework and performed our analysis with GenABEL [17] and ProbABEL [18], which uses the kinship matrix based on the pedigree structure.

Population stratification is known to create spurious associations if not properly controlled. The common practice in GWAS has been to control for stratification by using a number of top PCs of the GWAS markers as covariates in the association analysis. However, the optimal number of PCs has not been well established even for common-variant analysis, as shown by Peloso and Lunetta [19]. Therefore, to account for population stratification in our AA subjects, we used a varying number of the leading PCs as covariates in our analysis: no PC, the top 2 PCs and the top 10 PCs. Although the impact of population stratification on the analysis of rare variants has shown to be more subtle and complex [20-23], several papers have demonstrated advantages of using PCs derived from common variants (with MAF $\geq 5 \%$ ) [24-27]. Therefore, we computed PCs derived from 30,164 common variants in our
ExomeChip data. The genomic inflation factor $\lambda$ was calculated as the ratio of the observed median $\chi^{2}$ value to the expected median $\chi^{2}$ value.

\section{Results}

We performed a single-variant analysis and the 4 sets of genome-wide gene-based analysis using 79,578 lowfrequency variants: Burden-1, Burden-5, famSKAT-1, and famSKAT-5. For a varying number of PCs, the genomic inflation factors $\lambda$ are shown in table 1 . The singlevariant analysis exhibited no genomic inflation, whether PCs were included or not (with all $\lambda \leq 1.002$ ). Likewise, the burden tests did not exhibit much inflation, with 2 PCs leading to the lowest inflation $(\lambda=1.025$ and 1.006 for Burden-1 and Burden-5, respectively). In contrast, the SKAT analyses exhibited modest inflation, with no PCs leading to the lowest inflation $(\lambda=1.075$ and 1.094 for 


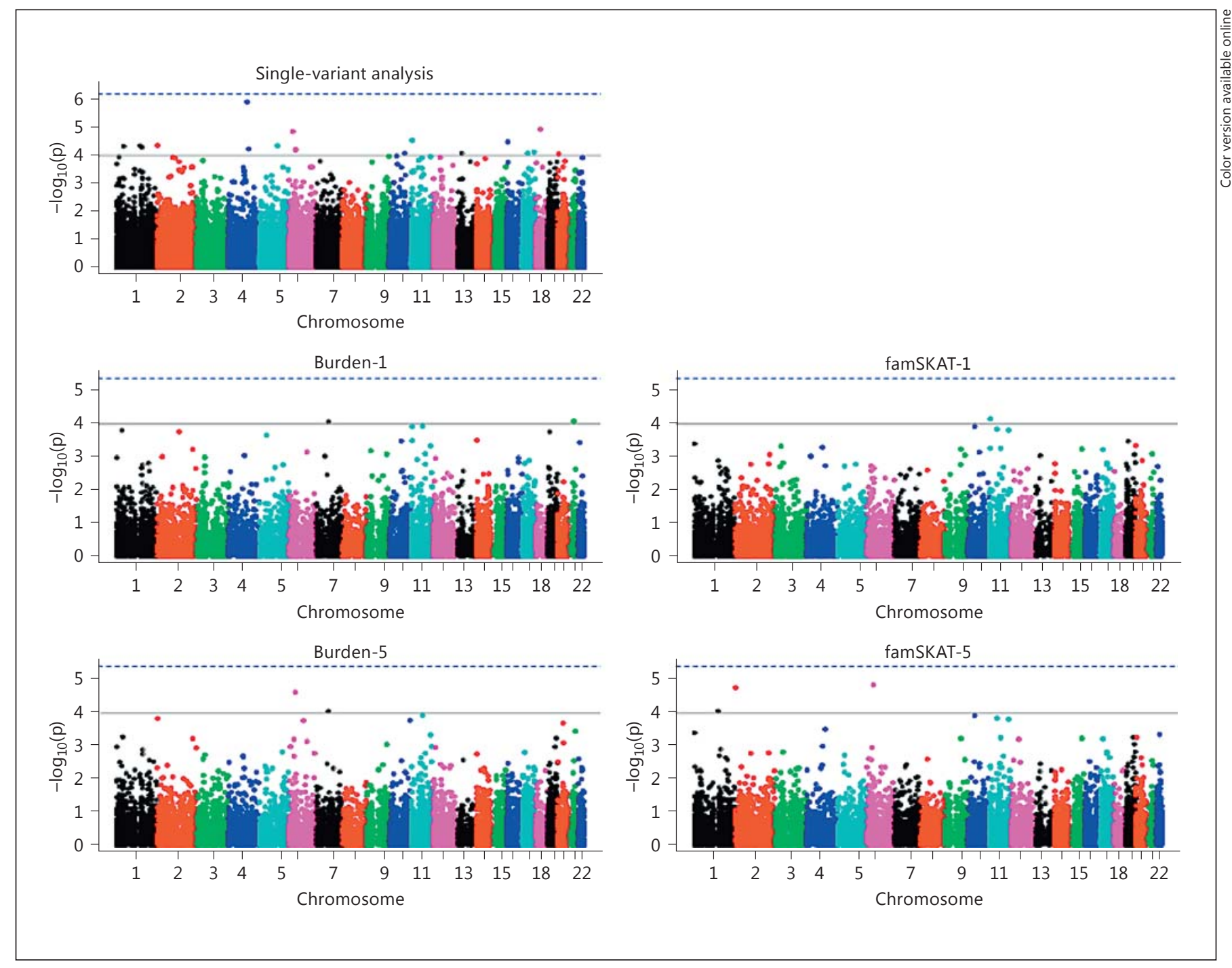

Fig. 1. Manhattan plots of the single-variant analysis and the 4 gene-level analysis options (Burden-1, Burden-5, famSKAT-1, and famSKAT-5). For the single-variant analysis, the $-\log _{10}(\mathrm{p})$ of each SNP was plotted at the chromosomal location of the SNP. For each

famSKAT-1 and famSKAT-5, respectively). Based on table 1 and also because our subjects were of African ancestry, we decided to present the results using 2 PCs. Quantile-quantile plots are shown in online supplementary figure 1 (for all online suppl. material, see www. karger.com/doi/10.1159/000375373).

Manhattan plots for the single-variant analysis and the 4 sets of gene-based analysis using 2 PCs are shown in figure 1. The genome-wide Bonferroni-corrected threshold was $6.4 \times 10^{-7}(=0.05 / 79,578)$ for the singlevariant analysis. The gene-based tests with MAF cutoffs

gene-level analysis, the $-\log _{10}(\mathrm{p})$ of each gene was plotted at the chromosomal location of the first SNP of the gene. These results are based on using 2 PCs.

of 1 and 5\% included 11,958 and 12,895 genes, respectively; the corresponding Bonferroni-corrected thresholds were $4.2 \times 10^{-6}$ and $3.9 \times 10^{-6}$, respectively. Table 2 presents the top 11 low-frequency variants from our single-variant analysis (with $\mathrm{p}<6.0 \times 10^{-5}$ ). Table 3 shows the top 16 genes from the 4 gene-based results (with $\mathrm{p}<$ $2.0 \times 10^{-4}$ ). We found no genome-wide significant results based on the Bonferroni-corrected threshold. However, we found that the gene-based methods often provided lower $\mathrm{p}$ values than the single-variant approach, as shown in table 3. 
We found a modest consistency across the 4 genebased analysis options. The results for the burden test and famSKAT test within each MAF threshold were moderately correlated (Spearman correlation $=0.55$ ) . The MAF thresholds (1 and 5\%) made more difference for the burden tests than the famSKAT tests (Spearman correlation $=0.50$ and 0.72 , respectively). The scatterplots of pairwise $-\log _{10}(\mathrm{p})$ values across the 4 gene-based analysis options are presented in online supplementary figure 2 .

We found that all 16 top genes in table 3 indeed had supporting evidence from multiple gene-based methods. To further evaluate the relative performance of each gene-based method, we classified the genes in table 3 into 4 groups. Note that the low-frequency variants (with $1 \% \leq \mathrm{MAF}<5 \%$ ) are presented in parenthesis in the 'Direction of single-variant association' column. For genes in group 1, the Burden-1 approach provided the strongest evidence; at these genes, rare variants had mostly unidirectional effects. We found that famSKAT also provided corroborating evidence for some of those genes (notably NSUN5 and FGF4), which is assuring. When the low-frequency variants had the opposite effects as compared to rare variants (as for OR52H1), the Burden-5 approach provided much weaker evidence than Burden-1. In contrast, when the low-frequency variants had the same effects as rare variants, the Burden-5 approach provided stronger evidence than Burden-1, as shown in group 2. We found that famSKAT indeed performed better on genes with multidirectional variants, as shown in groups 3 and 4 (notably HPX).

Some genes in table 3 had the most strongly associated single variant with the same magnitude of $p$ value as the gene-level results. To assess if the signal from the genelevel analyses is primarily driven by 1 single variant or aggregated evidence from multiple low-frequency variants, we performed a conditional analysis excluding this most strongly associated variant and using it as an additional covariate. Online supplementary table 1 provides the $\mathrm{p}$ values obtained by our conditional analysis. The signal for DOPEY2 appears to reflect aggregate evidence from multiple low-frequency variants. Five other genes, including NSUN5, also have a hint of aggregated effects, whereas the remaining genes have no significant conditional $p$ values, indicating their signals are mostly driven by one variant.

To improve the selection of variants to be included in the gene-level tests, we also considered functional annotation information. It turned out that most $(90,875$ out of

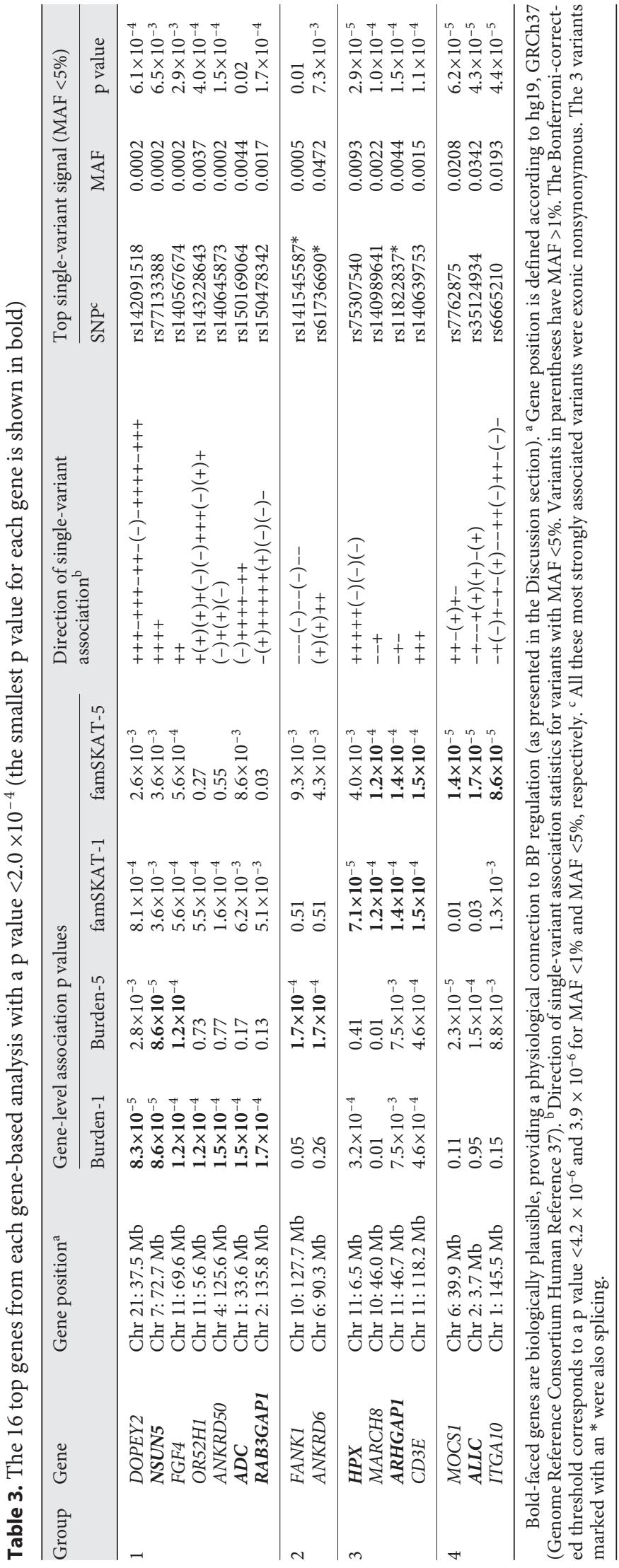

Sung et al. 
94,595) low-frequency variants were nonsynonymous, stop-gain/loss, splice, or missense variants. Therefore, when we repeated all 4 gene-level analyses with these functional variants only, the results were very similar (Spearman correlation $=0.97$ ) as shown in the first row of online supplementary figure 3 . Roughly half $(\mathrm{n}=$ 41,393 ) of the low-frequency variants were stop-gain/ loss, splice or missense variants and predicted to be damaging by 2 of the 4 algorithms in dbNSFP (Mutation Taster, Polyphen HDIV, SIFT or LRT). We further performed the 4 gene-level analyses with these damaging variants and found a subset of genes with lower $\mathrm{p}$ values (shown in the second row of online suppl. fig. 3). The most notable one was MOCS1, where the Burden- 5 result was enhanced $\left(\mathrm{p}=4.5 \times 10^{-6}\right)$. Our results illustrate that excluding neutral variants may help to improve the power of gene-level tests.

Although our focus was on low-frequency variants, we also performed a single-variant analysis on common variants. Among 30,164 common variants, our strongest signal was observed at rs11711441 on chromosome 3 (with $\left.\mathrm{p}=1.7 \times 10^{-5}\right)$. Out of 60 known BP variants identified by 9 articles [3, 28-35], only 13 were available in our ExomeChip data. Among these 13 known variants, the smallest $\mathrm{p}$ value from our analysis was observed at rs3184504 in SB2B3 (with $\mathrm{p}=0.0029$ ). The effect had the same direction as that reported by the International Consortium for Blood Pressure Genome-Wide Association Studies [3], but with lower MAF (0.057). Our results may not be a surprise given the limited number of common variants in the ExomeChip data.

\section{Discussion}

We performed an analysis of SBP using 79,578 rare and low-frequency variants from the Illumina exome array genotyped in 2,045 HyperGEN AAs. While neither the gene-based analyses nor the single-variant analysis produced genome-wide significant results, the most promising genes from each of the 5 methods had supporting evidence from some of the other gene-based methods. Of the genes for which the burden approach provided strongest evidence, 3 genes are of particular interest as potential BP candidate genes: NSUN5 (NOP2/Sun domain family), $A D C$ (arginine decarboxylase) and RAB3GAP1 (RAB3 GTPase-activating protein subunit 1). All 3 genes had corroborating evidence from SKAT as well. As the burden approach provided more significant evidence, the SNP effects in these genes are likely ho- mogeneous. NSUN5 is shown to be deleted in WilliamsBeuren syndrome [36], a multi-system developmental disorder whose symptoms include hypertension [37]. $A D C$ contains a conformational change-inducing missense mutation (substituting a polar amino acid, serine, for a nonpolar one, alanine) that has been previously associated with SBP [38]. RAB3GAP1 has been previously identified to be associated with sudden cardiac death in the MetaboChip study [39].

Of the genes with strong evidence from famSKAT analysis, 3 have potential connections to BP: $H P X(\mathrm{He}-$ mopexin), ARHGAP1 (Rho GTPase-activating protein 1 ) and $A L L C$ (allantoicase). HPX is a plasma glycoprotein that transports heme to the liver, and it is a major $\mathrm{BP}$ candidate gene owing to its role in regulating the expression of the angiotensin II type I receptor AGTR1 and modulating sensitivity to angiotensin [40] as well as its identification as a risk factor for late renal graft failure [41]. ARHGAP1 is a guanosine-triphosphatemetabolizing enzyme that plays a role in the regulation of tubule formation, an important early step in angiogenesis. ALLC is involved in the uric acid degradation pathway; uric acid levels have been associated with $\mathrm{BP}$ [42].

The single-variant analysis provided evidence for 2 additional potential candidate genes: ANK2 (ankyrin 2) and TRPC3 (transient receptor potential cation channel). ANK2 is a member of the ankyrin family of proteins and is required for the targeting and stability of the $\mathrm{Na}^{+} / \mathrm{Ca}^{2+}$ exchanger in cardiomyocytes. Mutations in this gene lead to long-QT syndrome and cardiac arrhythmia [43]. ANK2 has been located in a 1-lod support interval linked to resting heart rate [44]. TRPC3 is a renal $\mathrm{Ca}^{2+}$ channel, expressed in principal cells of the collecting duct. The expression levels of TRPC 3 have been associated with systolic hypertension in humans [45] and are higher in spontaneously hypertensive rats than in normotensive Wistar-Kyoto rats [46].

To evaluate the impact of population stratification on the analysis of rare variants, we used a varying number of leading PCs as covariates in our analysis. We found that type I error rates were well controlled for the single-variant and burden approaches, whereas the famSKAT approach exhibited modest inflation, as also noted by the famSKAT developers [14]. Furthermore, a recent article by Zawistowski et al. [23] also reports higher levels of inflation from the other non-burden tests including the Calpha test [12] and the original SKAT [13]. These nonburden tests have greater power to detect association in genes containing rare variants with heterogeneous effects 
but may be more vulnerable to population stratification. Given the lack of significant findings due to low power to detect rare variants, consistent findings from multiple gene-based methods including both burden and nonburden approaches with different thresholds are reassuring. These findings are compelling candidates for external validation.

\section{Acknowledgements}

We thank the anonymous reviewers for their constructive and insightful comments, which substantially improved the manuscript. The authors thank Maria Sosa for her lab assistance. The work was partly supported by multiple grants from the National Heart, Lung, and Blood Institute (NHLBI), National Institutes of Health (NIH): HL086694, HL111249, HL055673, and HL121091.

\section{References}

1 Newton-Cheh C, Johnson T, Gateva V, et al: Genome-wide association study identifies eight loci associated with blood pressure. Nat Genet 2009;41:666-676.

2 Levy D, Ehret GB, Rice K, et al: Genome-wide association study of blood pressure and hypertension. Nat Genet 2009;41:677-687.

3 International Consortium for Blood Pressure Genome-Wide Association Studies; Ehret GB, Munroe PB, Rice KM, et al: Genetic variants in novel pathways influence blood pressure and cardiovascular disease risk. Nature 2011;478:103-109.

4 Manolio TA, Collins FS, Cox NJ, et al: Finding the missing heritability of complex diseases. Nature 2009;461:747-753.

5 McCarthy MI, Hirschhorn JN: Genome-wide association studies: potential next steps on a genetic journey. Hum Mol Genet 2008; 17(R2):R156-R165.

6 Morgenthaler S, Thilly WG: A strategy to discover genes that carry multi-allelic or monoallelic risk for common diseases: a cohort allelic sums test (CAST). Mutat Res 2007;615: 28-56.

7 Li B, Leal SM: Methods for detecting associations with rare variants for common diseases: application to analysis of sequence data. Am J Hum Genet 2008;83:311-321.

8 Madsen BE, Browning SR: A groupwise association test for rare mutations using a weighted sum statistic. PLoS Genet 2009;5:e1000384.

9 Morris AP, Zeggini E: An evaluation of statistical approaches to rare variant analysis in genetic association studies. Genet Epidemiol 2010;34:188-193.

10 Cohen JC, Pertsemlidis A, Fahmi S, Esmail S, Vega GL, Grundy SM, Hobbs HH: Multiple rare variants in NPC1L1 associated with reduced sterol absorption and plasma low-density lipoprotein levels. Proc Natl Acad Sci USA 2006;103:1810-1815.

11 Ionita-Laza I, Buxbaum JD, Laird NM, Lange C: A new testing strategy to identify rare variants with either risk or protective effect on disease. PLoS Genet 2011;7:e1001289.

12 Neale BM, Rivas MA, Voight BF, Altshuler D, Devlin B, Orho-Melander M, Kathiresan S, Purcell SM, Roeder K, Daly MJ: Testing for an unusual distribution of rare variants. PLoS Genet 2011;7:e1001322.

13 Wu MC, Lee S, Cai T, Li Y, Boehnke M, Lin $\mathrm{X}$ : Rare-variant association testing for sequencing data with the sequence kernel association test. Am J Hum Genet 2011;89:82-93.

14 Chen H, Meigs JB, Dupuis J: Sequence kernel association test for quantitative traits in family samples. Genet Epidemiol 2013;37:196204.

15 Williams RR, Rao DC, Ellison RC, et al: NHLBI family blood pressure program: methodology and recruitment in the HyperGEN network. Hypertension genetic epidemiology network. Ann Epidemiol 2000;10:389-400.

16 Psaty BM, O’Donnell CJ, Gudnason V, et al: Cohorts for Heart and Aging Research in Genomic Epidemiology (CHARGE) Consortium: design of prospective meta-analyses of genome-wide association studies from 5 cohorts. Circ Cardiovasc Genet 2009;2:7380.

17 Aulchenko YS, Ripke S, Isaacs A, van Duijn CM: GenABEL: an R library for genome-wide association analysis. Bioinformatics 2007;23: 1294-1296.

18 Aulchenko YS, Struchalin MV, van Duijn CM: ProbABEL package for genome-wide association analysis of imputed data. BMC Bioinformatics 2010;11:134.

19 Peloso GM, Lunetta KL: Choice of population structure informative principal components for adjustment in a case-control study. BMC Genet 2011;12:64.

20 Mathieson I, McVean G: Differential confounding of rare and common variants in spatially structured populations. Nat Genet 2012; 44:243-246

21 Jiang Y, Epstein MP, Conneely KN: Assessing the impact of population stratification on association studies of rare variation. Hum Hered 2013;76:28-35.

22 O'Connor TD, Kiezun A, Bamshad M, Rich SS, Smith JD, Turner E; NHLBIGO Exome Sequencing Project; ESP Population Genetics, Statistical Analysis Working Group, Leal SM, Akey JM: Fine-scale patterns of population stratification confound rare variant association tests. PLoS One 2013;8:e65834.
23 Zawistowski M, Reppell M, Wegmann D, St Jean PL, Ehm MG, Nelson MR, Novembre J, Zollner S: Analysis of rare variant population structure in Europeans explains differential stratification of gene-based tests. Eur J Hum Genet 2014;22:1137-1144.

24 Purcell S, Neale B, Todd-Brown K, et al: PLINK: a tool set for whole-genome association and population-based linkage analyses. Am J Hum Genet 2007;81:559-575.

25 He H, Zhang X, Ding L, Baye TM, Kurowski BG, Martin LJ: Effect of population stratification analysis on false-positive rates for common and rare variants. BMC Proc 2011; 5(suppl 9):S116.

26 Babron MC, de Tayrac M, Rutledge DN, Zeggini E, Genin E: Rare and low frequency variant stratification in the UK population: description and impact on association tests. PLoS One 2012;7:e46519.

27 Price AL, Zaitlen NA, Reich D, Patterson N: New approaches to population stratification in genome-wide association studies. Nat Rev Genet 2010;11:459-463.

28 Johnson T, Gaunt TR, Newhouse SJ, et al: Blood pressure loci identified with a genecentric array. Am J Hum Genet 2011;89:688700 .

29 Padmanabhan S, Melander O, Johnson T, et al: Genome-wide association study of blood pressure extremes identifies variant near UMOD associated with hypertension. PLoS Genet 2010;6:e1001177.

30 Kato N, Takeuchi F, Tabara Y, et al: Metaanalysis of genome-wide association studies identifies common variants associated with blood pressure variation in east Asians. Nat Genet 2011;43:531-538.

31 Wain LV, Verwoert GC, O'Reilly PF, et al: Genome-wide association study identifies six new loci influencing pulse pressure and mean arterial pressure. Nat Genet 2011;43:10051011.

32 Franceschini N, Fox E, Zhang Z, et al: Genome-wide association analysis of bloodpressure traits in African-ancestry individuals reveals common associated genes in African and non-African populations. Am J Hum Genet 2013;93:545-554. 
33 Ganesh SK, Chasman DI, Larson MG, et al: Effects of long-term averaging of quantitative blood pressure traits on the detection of genetic associations. Am J Hum Genet 2014;95: 49-65.

34 Tragante V, Barnes MR, Ganesh SK, et al: Gene-centric meta-analysis in 87,736 individuals of European ancestry identifies multiple blood-pressure-related loci. Am J Hum Genet 2014;94:349-360.

35 Simino J, Shi G, Bis JC, et al: Gene-age interactions in blood pressure regulation: a largescale investigation with the CHARGE, Global BPgen, and ICBP Consortia. Am J Hum Genet 2014;95:24-38.

36 Doll A, Grzeschik KH: Characterization of two novel genes, WBSCR20 and WBSCR22, deleted in Williams-Beuren syndrome. Cytogenet Cell Genet 2001;95:20-27.

37 Schubert C: The genomic basis of the Williams-Beuren syndrome. Cell Mol Life Sci 2009;66:1178-1197.
38 Hong KW, Jin HS, Lim JE, et al: Non-synonymous single-nucleotide polymorphisms associated with blood pressure and hypertension. J Hum Hypertens 2010;24:763-774.

39 Huertas-Vazquez A, Nelson CP, Guo X, et al: Novel loci associated with increased risk of sudden cardiac death in the context of coronary artery disease. PLoS One 2013;8:e59905.

40 Krikken JA, Lely AT, Bakker SJ, Borghuis T, Faas MM, van Goor H, Navis G, Bakker WW: Hemopexin activity is associated with angiotensin II responsiveness in humans. J Hypertens 2013;31:537-541, discussion 542.

41 Krikken JA, van Ree RM, Klooster A, et al: High plasma hemopexin activity is an independent risk factor for late graft failure in renal transplant recipients. Transpl Int 2010;23: 805-812.

42 Loeffler LF, Navas-Acien A, Brady TM, Miller ER 3rd, Fadrowski JJ: Uric acid level and elevated blood pressure in US adolescents: $\mathrm{Na}$ tional Health and Nutrition Examination Survey, 1999-2006. Hypertension 2012;59: 811-817.
43 Sedlacek K, Stark K, Cunha SR, et al: Common genetic variants in ANK2 modulate QT interval: results from the KORA study. Circ Cardiovasc Genet 2008;1:93-99.

44 Martin LJ, Comuzzie AG, Sonnenberg GE, Myklebust J, James R, Marks J, Blangero J, Kissebah AH: Major quantitative trait locus for resting heart rate maps to a region on chromosome 4. Hypertension 2004;43:11461151.

45 Thilo F, Baumunk D, Krause H, Schrader M, Miller K, Loddenkemper C, Zakrzewicz A, Krueger K, Zidek W, Tepel M: Transient receptor potential canonical type 3 channels and blood pressure in humans. J Hypertens 2009;27:1217-1223.

46 Liu D, Yang D, He H, et al: Increased transient receptor potential canonical type 3 channels in vasculature from hypertensive rats. Hypertension 2009;53:70-76. 